\title{
Non-Linear Analysis: Music and Human Emotions
}

\author{
Juan $\mathrm{He}^{1, \mathrm{a}}$, Guangyuan Liu ${ }^{1, *}$, Wanhui Wen ${ }^{1, \mathrm{~b}}$ and Min Peng ${ }^{1, \mathrm{c}}$ \\ ${ }^{1}$ Southwest University, Chongqing 400715, China \\ ( ${ }^{a}$ xinandx, ${ }^{*}$ liugy, ${ }^{b}$ cwenwanh, ${ }^{c}$ peng2014m)@swu.edu.cn
}

Keywords: Music, Human Emotions, HRV, LLE

\begin{abstract}
The relationship between music and human emotions is a very interesting area. But how exactly music affects human emotions and what the valid parameters needed to classify the pattern is also an open question. In this paper, we consider the effect of four different types of music on human emotions through the nonlinear analysis of Heart rate variability (HRV). The t-test shows that the Largest lyapunov exponent (LLE) of HRV acquired from subjects with or without music stimulation is significantly different ( $p<0.01$, Cohen's $d>0.8$ ). More importantly, the LLE of HRV is also influenced by different types of music respectively, and then we applied LLE as the affective features to recognize the subjects' emotional state which induced by specific types of music. An overall correct rate of 84 percent for quinary classification of amusement, excitement, sadness, fear and the baseline state by SVM classifier are obtained.
\end{abstract}

\section{Introduction}

Affective computing has got widespread attention at the beginning of the development, and has become the research hotspot of arificial intelligence. Make machine to possesses emotion and to get the ability to identfy human emtion, has important research significane of realizing the harmonious human-computer interaction[1]. In this paper, music is used to evokes the participants' corresponding emotion, and ECG signal is real-time collected. Through the analysis of emotional ECG siganl to let machine have the ability wo recognize human emotion.

Despite whether listeners realize that their emotions evoked by music, some researches expanded the study on different types of music how to effect the human physiological status since Picard et al. [1] put forward that emotion recognition based on physiological signals is an effective method. Nyklícek et al. [2] observed changes in respiratory sinus arrhythmia (RSA) and cardiac interbeat intervals (IBI) of $26 \mathrm{Ss}$ at the onset of different types of music and demonstrated that the emotion categories induce by music were significantly classified. Iwanaga et al. [3] has found that that excitative music decreased the activation of the parasympathetic nervous system. Kallinen [4] examined the psychophysiological (i.e., ECG, EDA, and EMG) responses to listening to different types of music and found that unpleasant music drawing more attention (i.e., longer IBIs, lower HR) than pleasant music. Because human physiological signals is objectivity and reliability [5], more and more researchers [6-11] used the physiological signal changes to study the effects of music on human emotional state. In their papers, the ECG signal is the most common used. But there is no reliable feature of the ECG signals to classify the emotional patterns induced bymusic.

As Largest lyapunov exponent (LLE) has been proved to be an effective feature in the field of non-linear feature extraction of signals [12], we consider the effect of four different types of music (amusement, excitement, sadness, and fear) on human emotions through the LLE analysis of Heart rate variability (HRV). In this work, the HRV data is acquired from 30 normal healthy subjects' ECG signals which induced by these four different types of music. The t-test is used to detect the changes of LLE in physiological signals under different types of music. And then, the LLE affective features applied to a SVM to distinguish relaxation, excitement, sadness, fear and the baseline state. 


\section{Acquisition of affective data}

The Subjects and Emotion Elicitation Materials. In our experiment, 30 healthy subjects (aged 19-21 yrs) have been recruited from the freshmen. They have reported no recent history of medication, academic examination or psychological problems.

According to the music classification principles in [13], four types of music materials which can respectively arouse amusement, excitement, fear and sadness has been selected and applied in the experiment, and each type of music materials consists of three pieces of music works.

Data Acquisition .The experiment was carried out in a quiet lab room with adjustable light and suitable temperature. MP150, a multi-channel bio-signal recorder made by the Biopac Company, is used for data acquisition, and ECG signal is acquired at a sampling frequency of $400 \mathrm{~Hz}$. The experiment includes two rounds, and the average interval between them is about three weeks. The duration of each round is about 30 minutes, including 5 minutes of resting baseline, a session of music listening, 5 minutes of recovery, a session of another type of music listening and 5 minutes of recovery. Prior to the experiment, each subject has also signed an informed consent and filled out the Eysenck personality questionnaire which can reflect the personality traits of the subjects. In addition, they will be told the experimental instructions. The music in each session has been randomly selected from the three pieces of certain kind of music by the subjects. The experiment process is shown in Fig 1.

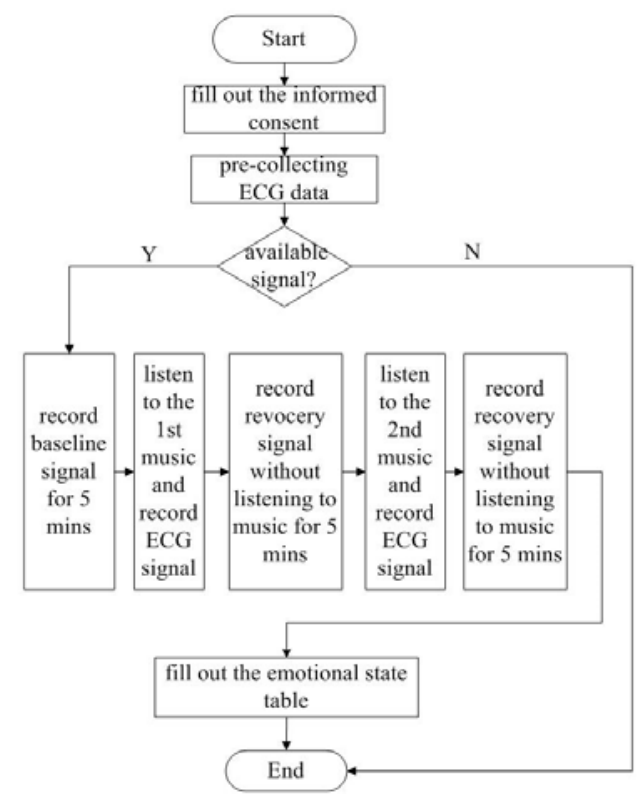

Fig. 1 Experimental process

Subjective emotional state questionnaire in this paper is referenced from an arousal-valence emotion model [14], as is shown in Fig 2. The horizontal axis is the valence, and the vertical axis denotes degree of arousal. After each session of the experiment, the subjects need to fill out in an emotional state questionnaire which maps the arousal-valence emotion space into quantitative emotional state, as is shown in Fig 3. Both sides of the horizontal axis are quantified into five levels (1-5), and the vertical axis is quantified into 10 levels (1-10), the higher the level, the more tension of the body. 


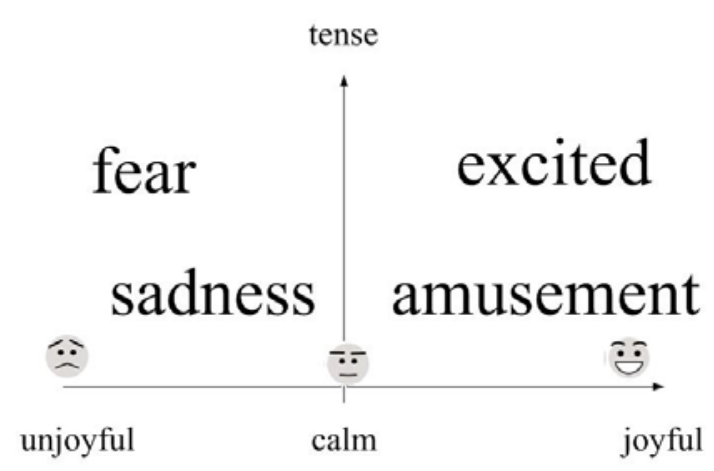

Fig. 2 Emotion model

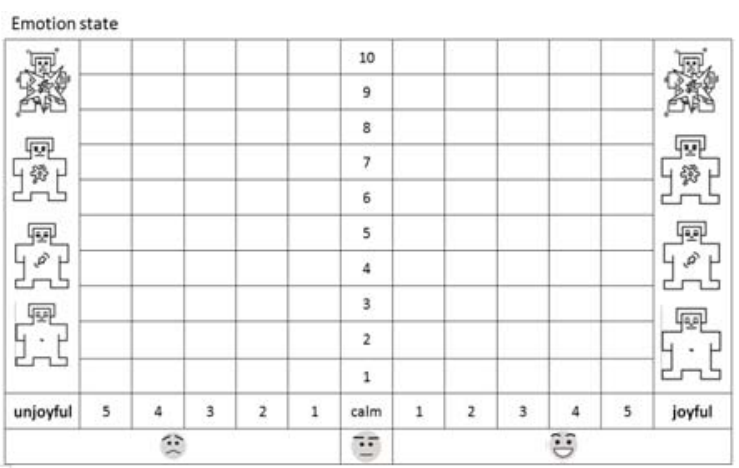

Fig. 3 Emotion state questionnaire

The valid ECG data which correspond to successfully aroused target emotions are selected based on the self-score of arousal level which should be no less than 3. Besides, the ECG data during obvious body motions have been excluded from the normal ECG data. Through the above data selection process, we can analyze the relationship between music feature space and emotional space.

\section{Method}

Data Preprocessing. The experiment collected ECG data of 30 subjects. As each of the subjects has taken part in both rounds of the experiment, there are $60 \mathrm{ECG}$ records collected before music listening and $120 \mathrm{ECG}$ records during music listening. After data screening, $97 \mathrm{ECG}$ records of emotional reaction were selected, 21 records for amusement music, 26 records for exciting music, 28 records for fear music, 22 records for sad music. Finally, the emotional ECG signal database has been set up by segmenting the middle 90 seconds data sample in each ECG record. Before analyzing the HRV signal, the ECG signal has been denoised to locate the R peaks of the QRS complex in the ECG signal. A 6-layer Mexican Hat wavelet decomposing has been applied to denoise the ECG signal in order to reduce the noise from EMG interference and baseline drift $[15,16]$.

Largest lyapunov exponent (LLE). As one of the most important indicators of systems' chaotic property judgment, LLE was increasing used by researchers for HRV signals' chaos property analysis. The LLE analysis of HRV shows the HRV signal's complexity which responses the non-linear relationship between the sympathetic nervous system and the parasympathetic nervous system. In this paper, we use the method which proposed by Rosenstein [17] about calculating largest Lyapunov exponents from small data sets. This method made full use of all data to get higher precision.

The small data sets method is shown below:

Considering a chaotic time series $\left\{x_{1}, x_{2} \cdots x_{n}\right\}$, the reconstructed trajectory $\mathbf{X}$ can be expressed as a matrix where each row is a phase-space vector. That is,

$$
\mathrm{X}=\left\{X_{i} \mid X_{i}=\left[x_{i}, X_{i+\tau}, \cdots, X_{i+(m-1) \tau}\right]^{T}, i=1,2, \cdots M\right\}
$$

where $\tau$ is the time delay and $m$ is the embedding dimension. Thus, $\mathbf{X}$ is an $\mathbf{M} \times \mathbf{N}$ matrix, and $\mathrm{M}=\mathrm{N}-(\mathrm{m}-1) \tau$. According to the theory proposed by Liebert and Schuster [18], the good approximation of $\tau$ is calculated by the autocorrelation function drops to $1-\frac{1}{e}$ of its initial value. The embedding dimension is usually estimated in accordance with Takens' theorem [19]. Firstly we need to calculate the correlation dimension $\mathrm{d}$, and then, based on $\mathrm{m}>2 \mathrm{~d}+1$ to determine $\mathrm{m}$.

After reconstructing the dynamics, the nearest neighbor $X_{\hat{j}}$ of each point $X_{j}$ on the reconstructed trajectory is expressed as 
$d_{j}(0)=\min _{X_{\hat{j}}}\left\|X_{j}-X_{\hat{j}}\right\|$

(2)

the $\|\cdot\|$ denotes the Euclidean norm and $d_{j}(0)$ is represent the initial distance from the $\mathrm{X}_{j}$ to its nearest neighbor. In order to avoid each point $\mathrm{X}_{j}$ and its nearest neighbor in the same trajectory, we have the additional constraint $|j-\hat{j}|>p$, where $\mathrm{p}$ is the mean period of the chaotic time series. For each point $\mathrm{X}_{j}$, calculate the distance of its nearest neighbor $\mathrm{X}_{\hat{j}}$ after $\mathrm{i}$-th discrete time steps, that is,

$d_{j}(i)=\min \left\|X_{j+i}-X_{\hat{j}+i}\right\|, \quad i=1,2, \cdots \min (M-j, M-\hat{j})$

(3)

Supposing the point $\mathrm{X}_{j}$ is in exponent dependence relationship with $\mathrm{X}_{\hat{\mathrm{j}}}$, written as

$d_{j}(i)=d_{j}(0) \times e^{\lambda_{1}(i \cdot \Delta t)}$

(4)

by taking the logarithm of both sides of Eq. (11), we obtain

$\ln d_{j}(i)=\ln d_{j}(0)+\lambda_{1} \cdot \Delta t \cdot i$

(5)

where $\Delta t$ is the length of the trajectory evolution.

For each step i,

$y(i)=\frac{1}{M \times \Delta t} \sum_{j=1}^{M} \ln d_{j}(i)$

(6)

Finally, the Largest lyapunov exponent is easily and accurately calculated using a least-squares fit to the $y(i)$.

\section{Result}

To test whether the LLE features of HRV at the baseline durations are significantly different from those of the music listening durations, Student's $t$ test and Cohen's $d$ test are used. The results are shown in Table 1.

Table 1 Statistic test of LLE between the baseline status and the music listening status

\begin{tabular}{ccccc}
\hline Calm/music & Calm/amusement & Calm/excitement & Calm/sadness & Calm/fear \\
\hline \multirow{2}{*}{ Lyapnov } & $\mathrm{P} \leq 0.006$ & $\mathrm{P} \leq 0.01$ & $\mathrm{P} \leq 0.01$ & $\mathrm{P} \leq 0.001$ \\
& Cohen's d=0.91 & Cohen's d=0.86 & Cohen's d=0.95 & Cohen's d=1.29
\end{tabular}

As is shown in Table 1, the result shown significant difference of LLE feature between the baseline and each of the four kinds of music listening. A boxplot of the LLE feature under baseline and four types of music is shown in Fig. 4

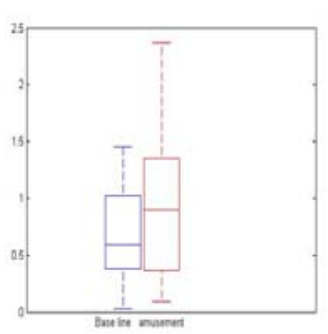

(a)

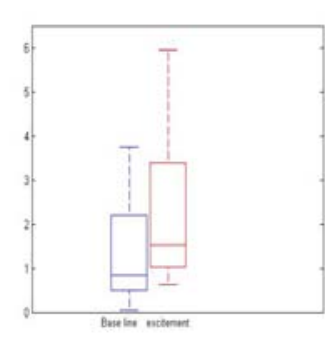

(b)

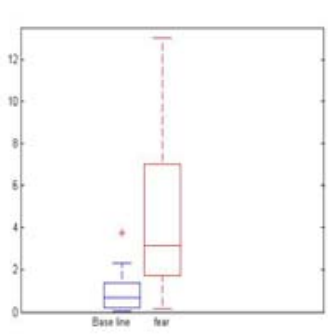

(c)

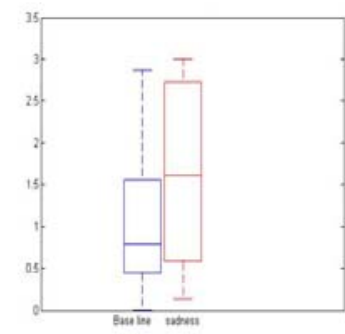

(d)

Fig. 4 The LLE features of the baseline status and music listening status

Each box in the chars includes six nodal points of a sequence, the high edge, $1^{\text {st }}$ Quartile, Median, $3^{\text {rd }}$ Quartile, low edge and the Outlier. The sign " + " in the chars stands the Outlier. The edges show 
the range of the data, the Quartilies and Median show the dispersion of the data.

Through by the Non-linear analysis of LLE, the above statistic tests verify that music definitely the impact on person's affective heartbeat response, and then influence the human emotional state. The next step is to test whether different type of music has different impact on the human emotional state. Table 2 shows the statistic test results of the LLE features, and the tests are performed between each pair of the four music types.

Table 2 Statistic tests of HRV features between pairs of different music types

\begin{tabular}{cccc}
\hline feature & amusement/excited & amusemen/fear & amusement/sadness \\
\hline Lyapunov & $\mathrm{P}<0.01$ & $\mathrm{P}<0.001$ & $\mathrm{P}<0.05$ \\
feature & Cohen's d=0.86 & Cohen's d $=1.30$ & Cohen's d=0.82 \\
Lyapunov & excited/fear & excited/sadness & fear/sadness \\
& $\mathrm{P}<0.05$ & $\mathrm{P}<0.01$ & $\mathrm{P}<0.005$ \\
Cohen's d=0.81 & Cohen's d $=0.88$ & Cohen's d=1.03 \\
\hline
\end{tabular}

As is shown in Table 2, the Largest lyapunov exponent has significant difference between each pair of the four music types. In addition, the types of amusement and fear music have maximum significant difference.

Figure 5 use box charts to show the LLE feature between each pair of music types, i.e. amusement vs. excitement, amusement vs. fear, amusement vs. sadness, excitement vs. fear, excitement vs. sadness and fear vs. sadness.

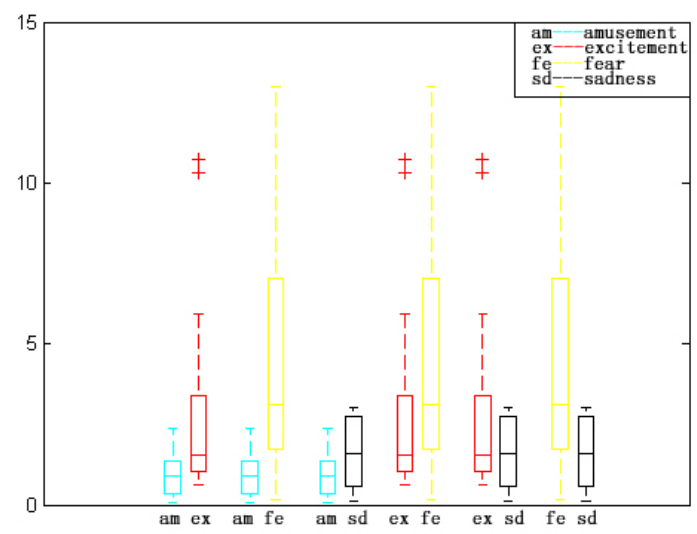

Fig. 5 The significant differences between two types of music-aroused affective heartbeat response

In order to further illustrate that different types of music has different effect on a person's emotional state, the SVM classifier [20] has been applied to classify the emotion pairs in Figure 6, and the binary classification accuracy of $82.3 \%, 90.2 \%, 79.2 \%, 78.6 \%, 88.2 \%$ and $89.3 \%$ for the emotion pairs in Figure 6 has been respectively obtained.

\section{Discussion}

According to the above results, different kinds of music exactly evokes people's different emotional pattern through affective heartbeat response. What's more, we proved that the LLE is an effective feature to classify those different emotional patterns induced by music. The mechanism of music affect person's emotional state may be that human auditory sensation converts the rhythm and melody into a response of the autonomic nerve activity.

It is worth noting that due to individual difference, the emotional feeling to a certain kind of music may be different among the subjects. The factors that cause these individual differences of music sensation include personal preference of music types, the familiar level to a certain kind of music, the background mood during the music listening, et al. 


\section{Acknowledgement}

This work was supported by the National Science Foundation of China (Grant No. 61472330 and No. 61103132). Author's brief introduction, Juan He, female, born in 1989, master graduate student, the main research direction for Affective Computing. Guangyuan Liu (Corresponding author), male, born in 1961, Ph.D., professor, main research direction for Affective Computing, Pattern Recognition, etc. E-mail: liugy@swu.edu.cn. WanHui Wen, female, born in 1981, PhD, associate professor, main research direction for Affective Computing, Pattern Recognition, etc. Min Peng, male, born in 1992, master graduate student, the main research direction for Affective Computing, Image Processing, etc.

\section{References}

[1] R. W. Picard. Affective Computing. the MIT Press, Massachusetts, (1997).

[2] Nyklícek I, Thayer J. F. and Van Doornen L. J. P. Cardiorespiratory differentiation of musically-induced emotions. Journal of Psychophysiology, Vol. 11(1997), p. 304-321.

[3] Iwanaga M, Kobayashi A, Kawasaki C. Heart rate variability with repetitive exposure to music. Biological psychology, Vol. 70(2005), p. 61-66.

[4] Kallinen, K. Emotion related psychophysiological responses to listening to music with eyes-open versus eyes-closed: Electrodermal (EDA), electrocardial (ECG), and electromyographic (EMG) measures. Paper presented at the International Conference on Music Perception \& Cognition, (2004).

[5] Kim K H, Bang S W, Kim S R. Emotion recognition system using short-term monitoring of physiological signals. Medical and biological engineering and computing, Vol.42(2004), p. 419-427.

[6] Sammler D, Grigutsch M, Fritz T, et al. Music and emotion: electrophysiological correlates of the processing of pleasant and unpleasant music. Psychophysiology, Vol.44(2007), p. 293-304.

[7] Kim J, André E. Emotion recognition based on physiological changes in music listening. Pattern Analysis and Machine Intelligence, IEEE Transactions on, Vol. 30(2008), p. 2067-2083.

[8] Orini M, Bailón R, Enk R, et al. A method for continuously assessing the autonomic response to music-induced emotions through HRV analysis. Medical \& biological engineering \& computing, Vol. 48(2010), p. 423-433.

[9] Cai J, Liu G, Hao M. The research on emotion recognition from ECG signal. Information Technology and Computer Science, 2009. ITCS 2009. International Conference on. IEEE, Vol. 1,(2009), p. 497-500.

[10] Ma C, Liu G. Feature extraction, feature selection and classification from electrocardiography to emotions.Computational Intelligence and Natural Computing,(2009). CINC'09. International Conference on. IEEE,Vol.1(2009), p. 190-193.

[11] Jaimovich J, Coghlan N, Knapp R B. Emotion in motion: A study of music and affective response. From Sounds to Music and Emotions. Springer Berlin Heidelberg, (2013),p. 19-43.

[12] LI G, ZHOU S, XU D. Computing the Largest Lyapunov Exponent from Time Series. Journal of Applied Sciences, Vol. 2(2003), p. 003.

[13] Thoma M V, Ryf S, Mopiyeddini C, et al. Emotion regulation through listening to music in eceryday situations. Cognition\&Emotion, Vol. 26(2012), p. 550-560.

[14] Thoma M V, Ryf S, Mopiyeddini C, et al. Emotion regulation through listening to music in eceryday situations. Cognition\&Emotion, Vol. 26(2012), p. 550-560.

[15] L. G. Gamero, M. Risk, J. F. Sobh, A. J. Ramirez and J. P. Saul. Heart rate variability analysis using Wavelet transfore [J]. Computers in Cardiology, 1996: $177-180$

[16] Carsten Meyer, Jose Fernandez Gavela, and Matthew Harris. Combining Algorithms in Automatic Detection of QRS Complexes in ECG Signals [J]. IEEE transactions on information 
technology in biomedicine, July, 2006 vol. 10, No. 3

[17] Rosenstein M. T, Collins J. J, De Luca C J. A practical method for calculating largest Lyapunov exponents from small data sets [J]. Physica D: Nonlinear Phenomena, 1993, 65(1): 117-134

[18] Liebert W, Pawelzik K, Schuster H G. Optimal embeddings of chaotic attractors from topological considerations [J]. EPL (Europhysics Letters), 1991, 14(6): 521

[19] Takens F. On the numerical determination of the dimension of an attractor [M]. Springer Berlin Heidelberg, 1985

[20] Keerthi S S, Shevade S K, Bhattacharyya C, et al. Improvements to Platt's SMO algorithm for SVM classifier design [J]. Neural Computation, 2001, 13(3): 637-649 